\title{
Latest developments in 3D analysis of geomaterials by Morpho+
}

\author{
Cnudde, Veerle ${ }^{* * * *}$, Vlassenbroeck, Jelle ${ }^{* * *}$, De Witte, Yoni** \\ Brabant, Loes ${ }^{* * *}$, Boone, Matthieu, ${ }^{* *}$, Dewanckele, Jan ${ }^{*, * *}$, Van \\ Hoorebeke, Luc ${ }^{* *}$, Jacobs, Patric ${ }^{*, * *}$. \\ * Department of Geology and Soil Science, Ghent University, Krijgslaan 281/S8, B- \\ 9000,Ghent, Belgium \\ veerle.cnudde@ugent.be \\ jan.dewanckele@ugent.be \\ patric.jacobs@ugent.be \\ ** Department of Subatomic and Radiation Physics, Ghent University, \\ Proeftuinstraat 86, B-9000 Ghent, Belgium \\ jelle.vlassenbroeck@ugent.be \\ yoni.dewitte@ugent.be \\ loes.brabant@ugent.be \\ matthieu.boone@ugent.be \\ luc.vanhoorebeke@ugent.be
}

\begin{abstract}
At the Centre for X-ray Tomography of the Ghent University (Belgium) (www.ugct.ugent.be) besides hardware development for high-resolution X-ray CT scanners, a lot of progress is being made in the field of $3 D$ analysis of the scanned samples. Morpho+ is a flexible $3 D$ analysis software which provides the necessary petrophysical parameters of the scanned samples in 3D. Although Morpho+ was originally designed to provide any kind of $3 D$ parameter, it contains some specific features especially designed for the analysis of geomaterial properties like porosity, partial porosity, pore-size distribution, grain size, grain orientation and surface determination. Additionally, the results of the $3 D$ analysis can be visualized which enables to understand and interpret the analysis results in a straightforward way. The complementarities between high-quality X-ray CT images and flexible $3 D$ software are opening up new gateways in the study of geomaterials.
\end{abstract}




\section{Introduction}

At the Centre for X-ray CT of the Ghent University in Belgium (UGCT, www.ugct.ugent.be) there is a continuous drive for performing X-ray tomography scans at the highest quality and highest resolution with laboratory equipment. The combination of X-ray CT and 3D visualisation is a powerful technique which allows to look inside the samples in a non-destructive way. Although $3 \mathrm{D}$ visualisation is a powerful tool to investigate the sample after reconstruction, it has its limitations. While large and apparent structures can be rendered and their shapes and relative sizes can be assessed qualitatively, a lot of information remains hidden. To extract quantitative information, the volume has to be analysed using appropriate computer algorithms. Since a complete volume of linear attenuation coefficients can be obtained from X-ray computed tomography, the possibilities to analyse the resulting data are extensive. While a single cross-section can already reveal a lot of useful information, the true power of X-ray tomography lies within the capability to extract quantitative information about internal and external three-dimensional structures. Therefore Morpho+, a flexible 3D analysis software package, was developed inhouse, in order to provide any kind of 3D parameter for any kind of scanned material.

\section{Morpho+}

Morpho+ is a new software package developed at the UGCT (Belgium) which provides 3D data of CT scans like porosity, pore-size distribution, grain orientation, sphericity, etc. The development of Morpho+ was initiated by the observation that the in-house developed Matlab analysis package $\mu$ CTanalySIS (Cnudde et al., 2004) showed several shortcomings like the lacking of user interface and advanced level of functionality. Although Morpho+ builds on some of the concepts used in $\mu$ CTanalySIS, it combines this with more performance, a more extensive set of analysis tools and an interactive and intuitive user interface. Due to the versatility of the applications at the UGCT, full control over the analysis process and a thorough understanding of the underlying algorithms is necessary was an essential requirement for this software. Additionally, the possibility to implement custom algorithms for specific applications was a prerequisite. These conditions had to be combined with a high computational performance and memory efficient code. In order to obtain the necessary user-friendly interface, performance and coding flexibility, Morpho+ was programmed in $\mathrm{C}++$ using the Qt巴 application framework.

The typical work-flow for the 3D analysis in Morpho+ will be illustrated on the dataset of a North Sea reservoir sandstone. This sandstone was scanned with the flexible micro CT set-up from UGCT. After scanning and reconstruction of the images, the different steps possible in Morpho+ in order to obtain 3D data are described. 


\subsection{Scanning conditions}

For this sandstone sample the micro CT scanner of UGCT was used. The transmission head (Feinfocus, FXE-160.51) was used with a tube voltage of $110 \mathrm{kV}$ and a tube current of $73 \mu \mathrm{A}$. The scan time was around 1 hours. The highest spatial resolution of this system, depending on the sample size, the spot size of the X-ray source and the resolution of the detector, is around $1 \mu \mathrm{m}$. For this set-up, with a geometrical magnification of 23, a resolution of $3.97 \mu \mathrm{m}$ was obtained in all directions. As detector, the Varian PaxScan 2520V (1880 x 1496 pixels) was used. 1200 projections were taken over $360^{\circ}$, with an exposure time of $300 \mathrm{~ms}$ per frame and 5 frame averages were taken per projection. After reconstruction of the projections with the software package Octopus (Vlassenbroeck et al., 2007), a series of horizontal cross-sections (fig. 1a) was obtained, ready to be analysed.

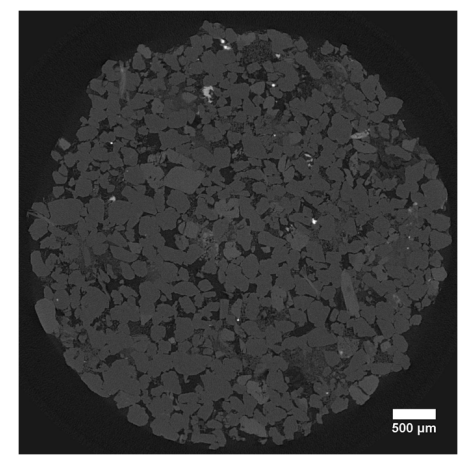

Figure 1. Reconstructed cross-section through a North Sea reservoir sandstone

\subsection{D analysis by Morpho+}

The 2D reconstructed images all contain information which is visualised in a greyscale representation inside the images. This grey sale is used for further 3D analysis in Morpho+. However, a greyscale volume is only a discrete representation of a real world object, which poses several limitations, like the fact that features smaller than the voxel size cannot be distinguished. Although only cross-sections of the analysed volume are shown in the following paragraphs, all analysis steps in Morpho+ are performed in 3D.

\subsubsection{Morpho+ analysis steps}

\subsubsection{Volume of interest selection}

When performing 3D analysis, first the volume of interest (VOI) needs to be selected in order to omit irrelevant data which can give wrong analysis results. For 
example, when analysing the porosity distribution in a geological sample, the air outside the sample should not be included in the analysis. For cylindrical or cuboid samples, a VOI can be selected by defining a circular or rectangular region in one cross-section and by propagating this selection over a number of cross-sections in the stack. Additionally, Morpho+ allows to rotate a cylindrical or cuboid VOI to compensate for sample tilt. Irregular shaped samples need a more intelligent approach for the selection of a VOI. In Morpho+ this is possible by using segmentation and binary operations in order to select the correct VOI.

\subsubsection{Segmentation and filtering techniques}

Segmentation algorithms are aimed at extracting structural features from the volume, like the pore network or the grains inside the sandstone. To facilitate the segmentation several noise filters are available in Morpho+, where each filter has its own unique properties in terms of noise reduction and the preservation of image sharpness. The median filter is a non-linear filter, where each voxel value is replaced by the median of its neighbouring voxels. This filter can reduce the spread and overlap of the distributions of the grey values of the different components inside an image, which makes it easier to segment the different components since it preserves straight edges, although sharp corners are rounded. The Gaussian filter is a linear filter, since each voxel of the volume is replaced by a linear combination of voxels within a kernel of size $\mathrm{N} \times \mathrm{N} \times \mathrm{N}$ centred around the voxel. This filter results however in a blurring effect around edges.

The bilateral filter is an extension of the Gaussian filter, where the actual greyvalues of the neighbouring voxels are taken into account. When a material voxel close to an edge is evaluated in the Gaussian filter, the neighbouring voxels containing the same material are processed in the same way as the voxels corresponding to air. The bilateral filter (Tomasi \& Manduchi, 1998) solves this problem by adapting the multiplication factors of the filter kernel based on the actual greyvalues of the neighbouring voxels.

After the filtering operations, the data is thresholded to separate the material of interest from the background. During this operation a binary volume is created and voxels are categorized as foreground voxels, when their greyscale value lies within a certain interval, and as background voxels, when they are outside of the interval. The threshold level can be calculated automatically in Morpho+ by using Otsu's method which assumes the volume is composed of two components, where each component shows a certain distribution of greyvalues in the histogram, and both distributions have an overlapping region (Otsu, 1979). When applied to real data, Otsu's method and other automatic thresholding techniques often fail, especially when the partial volume effect (the greyvalue of a voxel corresponds to the average linear attenuation coefficient of the different materials inside the voxel) play a significant role. Besides automatic thresholding, several classes of manual thresholding techniques exist in Morpho+ like single, which is sensitive to residual image noise, and dual thresholding, which uses two separate threshold values (or intervals) to reduce the sensitivity to residual image noise. In the scanned sandstone, besides the dense inclusions, the grains or the pore structure can easily be segmented by using the dual thresholding technique. 
Several binary operations, including opening, closing, removal of isolated foreground/background voxels and the filling of holes are implemented in Morpho+ which can help to remove or reduce the still remaining noise after thresholding. For our sample this was however not necessary.

\subsubsection{Labelling}

The next step in the analysis process is the labelling. The binary volume is therefore divided into several connected components called objects, where each object is assigned a unique value (colour code). This object labelling is used to detect each connected component of foreground voxels in the binary image and to assign a unique label to all voxels of that object. In Morpho+, a series of algorithms is used to detect the connected components (Roselfeld \& Pfaltz, 1966; Knuth, 1997). The labelling algorithm results in a 3D array of integer labels and a list of objects is extracted from this array. Each object can then be analysed separately, which adds a significant flexibility to the analysis process. Each object is characterised by its bounding box, the position of its bounding box in the original volume and the object voxels inside the bounding box. After the extraction of the list of objects, several operations are possible. Before proceeding with the other analysis steps, it is often interesting to apply the fill holes algorithm to the individual objects.

\subsubsection{Distance transform}

After the labelling algorithm, the Euclidean distance transform (Guan \& Ma, 1998; Delerue, 2001) is applied which serves several purposes in Morpho+. It can be used to separate objects which are initially connected, combined with the watershed segmentation, based on the algorithm by Vincent et al. (1991). Also, the maximum opening of an object, defined as the diameter of the maximum inscribed sphere which fits inside the object, can be extracted from its distance transform.

\subsubsection{Quantitative information}

After obtaining the distance transform, several parameters can be determined quantitatively in Morpho+. The total porosity can be obtained by dividing the total number of foreground voxels by the total number of voxels in the VOI. For a total analyzed volume of the scanned sandstone a total porosity of $13 \%$ was found. Since the total volume can be subdivided in several blocks in any direction (X, Y or Z) it is possible to evaluate the porosity per block. This way the porosity distribution in the sample can be extracted. The determination of the percentage of open and closed pores can also be distinguished. The open porosity is defined as the porosity of all border objects, while the closed porosity is composed of the isolated objects. Additionally, it is possible to extract information from each object. This data can be exported for each object and/or distributions can be extracted. The maximum opening and the total volume of each object can be determined. If we construct an equivalent sphere with the same total volume, the corresponding diameter is defined as the equivalent diameter. Based on the maximum opening and equivalent diameter, the sphericity $\mathrm{S}$ is defined as the ratio of both. This parameter gives a rough approximation of the shape of objects, since it expresses how much an object 
resembles a sphere $(S=1)$. Since Morpho+ can determine the orientation of the objects of interest, such as the grains inside the sandstone, it is possible to plot a stereoplot derived from the 3D analysis of the grains. Additionally, the Euler number, which expresses the multi connectivity of a volume and can be used to compare the connectivity of different (similar) samples, and the fractal dimension, determining how efficiently an object fills the space under consideration, as well as surface extraction, the determination of connections, and skeletonisation are also implemented in Morpho+ (Brabant, 2009). In the sandstone sample the Euler number of the pore network was -68929. A single value of the Euler number is difficult to interpret and these values are more relevant when comparing very similar rocks. Morpho+ also makes it possible to count the number of objects that are not connected to each other, so in this case the number of isolated pore networks. In the analysed sandstone sample 61038 isolated pore networks were identified. There is however always very few networks and the other ones are very small. Now it is possible to calculate the number of tunnels through the pore network. There are 140510 tunnels through the pore network of sample one, which illustrates that sample one has a high multi-connectedness because of the large amount of tunnels through the network and not because there are no isolated parts in the network. The fractal dimension of the grains of the sandstone sample is 2.769 .

The surface of the separate objects or the complete volume can also be extracted by applying the marching cubes algorithm. The resulting 3D mesh can be saved as an STL file and can be used in other software packages for visualisation and analysis purposes (for example, finite element simulations). The result is shown for the grains of the sandstone sample (fig. 2). It is also possible to calculate the surface based on the marching cube algorithm. The pore network within a volume of height $3.08 \mathrm{~mm}$, depth $2.01 \mathrm{~mm}$ and width $2.33 \mathrm{~mm}$ has a surface of $369 \mathrm{~mm}^{2}$ for the scanned sample.

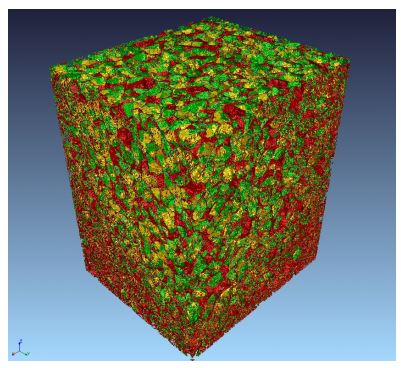

Figure 2. The visualization of grains of a sandstone sample. The outside of the grains is coloured in red, the inside in green en the dividing planes are coloured in yellow

\subsection{Data visualisation}

The processed volume can be visualized after each step in the analysis process. Since all analysis algorithms operate in three dimensions, cross-sections of the 
volume according to the different principal planes can be visualized. Each object can be relabelled (colour coded) based on a certain parameter, like for example the equivalent diameter where the blue objects have a small equivalent diameter and the red objects are the largest. To illustrate the different possibilities to process the object data, figure 3 illustrates the distribution of the equivalent diameter, sphericity, the orientation and the stereoplot of the analysed quartz grains inside the sandstone.

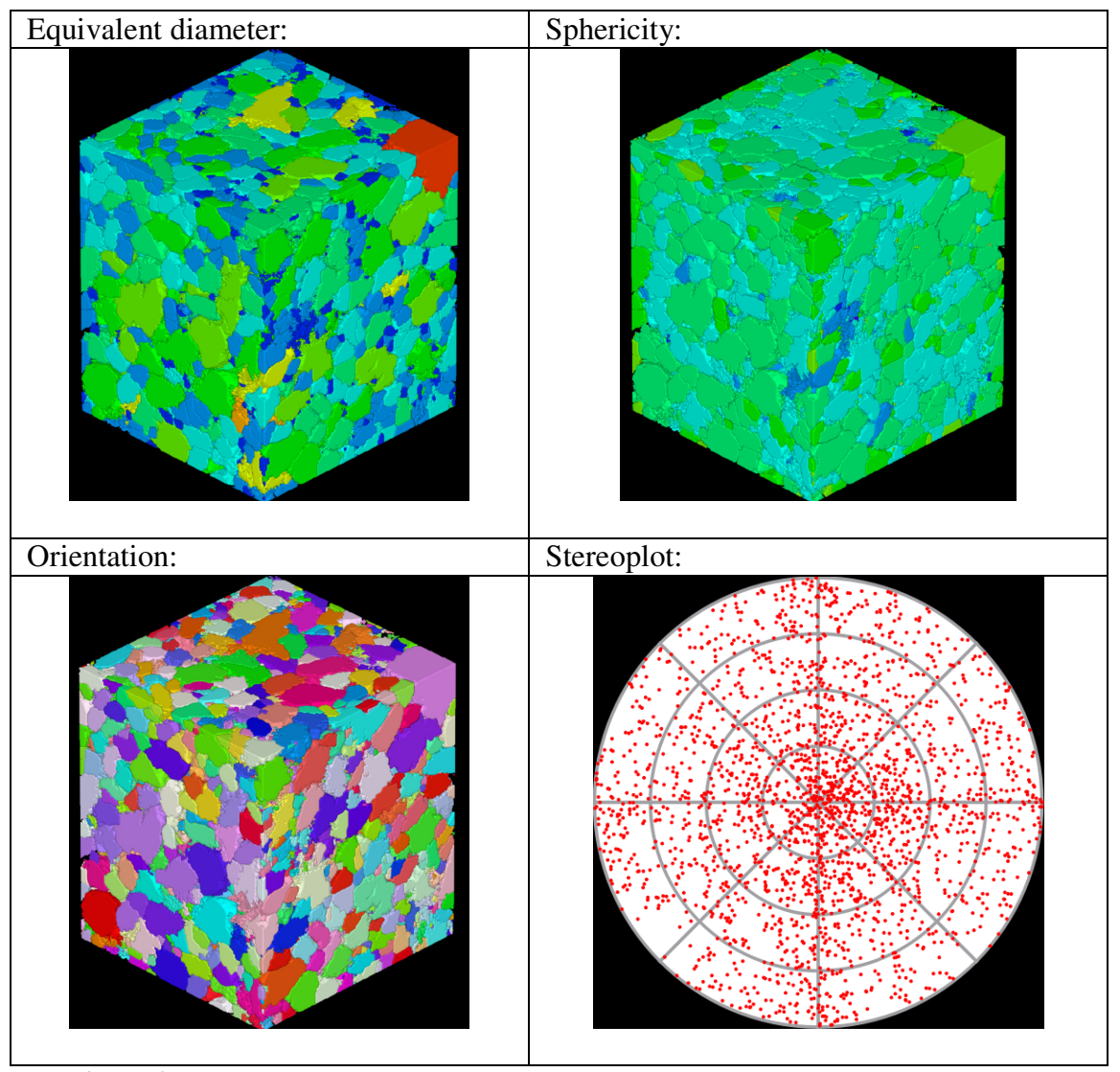

Figure 3. $3 D$ renderings of the colour-labeled images after $3 D$ analysis with Morpho+

\section{Conclusions}

Morpho+ contains some specific features especially designed for the analysis of geomaterial properties like porosity, partial porosity, pore-size distribution, grain size, grain orientation and surface determination. Additionally, the results of the 3D analysis can be visualized which enables to understand and interpret the analysis 
results in a straightforward way. The complementarities between high-quality X-ray CT images and flexible 3D software are opening up new gateways in the study of geomaterials.

Acknowledgements

The Fund for Scientific Research-Flanders (FWO) is acknowledged for the post-doc grant to V. Cnudde. The Institute for the Promotion of Innovation by Science and Technology in Flanders, Belgium is acknowledged for the PhD grant of J. Dewanckele.

\section{References}

Cnudde, V., Cnudde, J.P., Dupuis, C., Jacobs, P. X-ray micro-CT used for the localization of water repellents and consolidants inside natural building stones. Materials Characterization, vol. 53 no. 2-4, 2004, p. 259 - 271.

Tomasi, C., Manduchi, R., Bilateral Filtering for Gray and Color Images. Computer Vision, IEEE International Conference, 0:839, 1998.

Otsu, N. A threshold selection method from gray-level histograms. IEEE Transactions on Systems, Man and Cybernetics, vol. 9 no. 1, 1979, p. 62-66.

Rosenfeld, A., Pfaltz, J.L. Sequential Operations in Digital Picture Processing. J. $A C M$, vol. 13 no. 4, p. 471-494, 1966.

Knuth, D.E. Art of Computer Programming, Volume 1: Fundamental Algorithms (3rd Edition). Addison-Wesley Professional, July 1997.

Guan, W., Ma, S. A List-Processing Approach to Compute Voronoi Diagrams and the Euclidean Distance Transform. IEEE Transactions on Pattern Analysis and Machine Intelligence, vol. 20 no. 7, 1998, 757-761.

Delerue, J.F. Segmentation 3D, application l'extraction de réseaux de pores et la caractérisation hydrodynamique des sols. PhD thesis, Paris XI Orsay, 2001.

Vincent, L., Soille, P. Watersheds in digital spaces: an efficient algorithm based on immersion simulations., IEEE Transactions on Pattern Analysis and Machine Intelligence, vol. 13 no. 6, 1991, p. 583-598.

Brabant, L. Geavanceerde algoritmes voor 3D-analyse van micro-CT data. Master's thesis, Ghent University, Belgium, 2009.

Vlassenbroeck, J., Dierick, M., Masschaele, B., Cnudde, V., Van Hoorebeke, L., Jacobs, P. Software tools for quantification of X-ray microtomography at the UGCT. Nuclear Instruments and Methods in Physics Research Section A: Accelerators, Spectrometers, Detectors and Associated Equipment, 2007, 580(1):442-445. 